\title{
Microvascular inflammation is a risk factor in kidney transplant recipients with very late conversion from calcineurin inhibitor- based regimens to belatacept
}

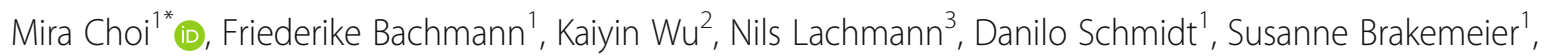
Michael Duerr ${ }^{1}$, Andreas Kahl ${ }^{1}$, Kai-Uwe Eckardt ${ }^{1}$, Klemens Budde $^{1}$ and Peter Nickel ${ }^{1}$

\begin{abstract}
Background: In de novo kidney transplant recipients (KTR) treatment with belatacept has been established as a comparable option as maintenance immunosuppression, preferably as a strategy to convert from calcineurin inhibitor (CNI)- to belatacept-based immunosuppression. Switch to belatacept demonstrated improved renal function in patients with CNI-induced nephrotoxicity, but risk of transplant rejection and the development of donor-specific antibodies (DSA) are still a matter of debate. Only few data are available in patients at increased immunological risk and late after transplantation.
\end{abstract}

Methods: We analyzed 30 long-term KTR (including 2 combined pancreas-KTR) converted from CNI to belatacept $>60$ months after transplantation with moderate to severe graft dysfunction (GFR $\leq 45 \mathrm{~mL} / \mathrm{min}$ ). Biopsies were classified according to the Banff 2015 criteria. Group differences were assessed in a univariate analysis using Mann Whitney $\mathrm{U}$ or Chi square test, respectively. Multivariate analysis of risk factors for treatment failure was performed using a binary logistic regression model including significant predictors from univariate analysis. Fifty-six KTR matched for donor and recipient characteristics were used as a control cohort remaining under CNI-treatment.

Results: Patient survival in belatacept cohort at 12/24 months was $96.7 \% / 90 \%$, overall graft survival was 76.7 and $60.0 \%$, while graft survival censored for death was $79.3 \% / 66.7 \%$. In patients with functioning grafts, median GFR improved from $22.5 \mathrm{~mL} / \mathrm{min}$ to $24.5 \mathrm{~mL} / \mathrm{min}$ at 24 months. Positivity for DSA at conversion was $46.7 \%$. From univariate analysis of risk factors for graft loss, GFR $<25 \mathrm{~mL} / \mathrm{min}(p=0.042)$ and Banff microvascular inflammation (MVI) sum score $\geq 2(p=$ 0.023 ) at conversion were significant at 24 months. In the analysis of risk factors for treatment failure, a MVI sum score $\geq 2$ was significant univariately $(p=0.023)$ and in a bivariate $(p=0.037)$ logistic regression at 12 months. DSA-positivity was neither associated with graft loss nor treatment failure. The control cohort had comparable graft survival outcomes at 24 months, albeit without increase of mean GFR in patients with functioning grafts ( $\Delta$ GFR of $-3.6 \pm 8.5 \mathrm{~mL} / \mathrm{min}$ ).

Conclusion: Rescue therapy with conversion to belatacept is feasible in patients with worsening renal function, even many years after transplantation. The benefit in patients with MVI and severe GFR impairment remains to be investigated.

Keywords: Belatacept, Allograft failure, Kidney transplantation, Calcineurin inhibitor toxicity

\footnotetext{
* Correspondence: mira.choi@charite.de

'Department of Nephrology and Medical Intensive Care, Charité Universitätsmedizin Berlin, Augustenburger Platz 1, 13353 Berlin, Germany

Full list of author information is available at the end of the article
}

(c) The Author(s). 2020 Open Access This article is licensed under a Creative Commons Attribution 4.0 International License, which permits use, sharing, adaptation, distribution and reproduction in any medium or format, as long as you give appropriate credit to the original author(s) and the source, provide a link to the Creative Commons licence, and indicate if changes were made. The images or other third party material in this article are included in the article's Creative Commons. licence, unless indicated otherwise in a credit line to the material. If material is not included in the article's Creative Commons licence and your intended use is not permitted by statutory regulation or exceeds the permitted use, you will need to obtain permission directly from the copyright holder. To view a copy of this licence, visit http://creativecommons.org/licenses/by/4.0/ The Creative Commons Public Domain Dedication waiver (http://creativecommons.org/publicdomain/zero/1.0/) applies to the data made available in this article, unless otherwise stated in a credit line to the data. 


\section{Background}

Long-term calcineurin inhibitor (CNI) exposure has been associated with numerous adverse effects such as nephrotoxicity, infections, hypertension, diabetes and dyslipidemia [1, 2]. Belatacept is a selective co-stimulation inhibitor that has been developed for CNI-free treatment. In de novo kidney transplant recipients treatment with belatacept has shown superior graft function and better patient and/or graft survival until 7 years post-transplant compared to cyclosporine despite increased early acute rejection rates [3-5]. Furthermore, belatacept use was associated with lower frequencies of patients developing donorspecific antibodies (DSA) [6]. While increased acute cellular rejection rates point to a lesser efficacy of belatacept compared to $\mathrm{CNI}$, reasons for the observed lower DSA rates might be an improved adherence to the intravenous belatacept regimen or superior B-cell control under belatacept. In fact, some studies suggested that belatacept may have regulatory effects on B cells $[7,8]$.

Several studies have evaluated risks and benefits of conversion from CNI-based treatment to belatacept [9-12]. However, few data are available on late (> 60 months posttransplant) conversion of patients with high immunological risk factors, such as DSA, after transplantation [13-16].

Here, we analyzed a cohort of kidney and combined kidney and pancreas transplant recipients at our center converted from CNI-based regimens to belatacept at a very late time point of $>60$ months after transplantation for predictive factors for graft loss or GFR deterioration after a period of 12 and 24 months. Patients were at increased immunological risk with a high percentage of $47 \%$ DSA-positivity at the time of conversion.

\section{Methods}

This retrospective study analyzed the outcome in all adult kidney and combined kidney and pancreas transplant patients with moderate to severe graft dysfunction (GFR $\leq 45 \mathrm{~mL} / \mathrm{min}$ ) that were converted from a CNI-based maintenance immunosuppressive regimen to belatacept at a very late stage of $>60$ months after last transplantation between 11/2012 and 11/2016. Chronic Kidney Disease Epidemiology Collaboration (CKD-EPI) formula was used to calculate estimate glomerular filtration rates. All patients were Epstein-Barr seropositive. At the time of conversion, belatacept was administered at a dose of $5 \mathrm{mg} / \mathrm{kg}$ intravenously on days 1, 15, 29, 43 and 57 in line with Rostaing et al. [12]. Thereafter, treatment was continued every 4 weeks. Due to CNI toxicity after long-term transplantation calcineurin inhibitor dose was tapered in a modified way as follows: to $50 \%$ the day after conversion (day 2), to $25 \%$ on day 15 and 0 on day 29 and thereafter. Immunosuppressive co-medication was continued. Biopsies were classified according to the Banff 2015 criteria [17]. Biopsies taken before publication of the Banff 2015 classification were scored retrospectively.

The primary outcome was treatment failure defined as renal graft loss or deterioration of GFR at 12 and 24 months compared to baseline GFR at the time of conversion. Treatment success was defined as stable GFR or improvement at 12 and 24 months. The following risk factors were analyzed for association with treatment failure at 12 and 24 months: biopsy scores, gender, donor age, living donation, patient age, conversion time after transplantation, body mass index (BMI), post-transplant diabetes mellitus, systolic and diastolic blood pressure, immunosuppressive regimen, history of any rejection prior to conversion, DSA positivity, eGFR and proteinuria at the time of conversion.

\section{Matched-pair analysis}

In order to generate a matched control group, a cohort of potential patients was identified using our web-based electronic patient record system "TBase" [18] in analogy to the belatacept treatment cohort. Patients were 1:2 matched for age ( \pm 5 years), donor age ( \pm 5 years), gender, immunosuppressive regimen with exclusion of mTOR therapy, GFR +/- $5 \mathrm{~mL} / \mathrm{min}, \mathrm{GFR} \leq 45$ between 2012 and 2016 and at start of observation period, and availability of renal transplant biopsies prior to observation.

\section{Data analysis}

IBM SPSS statistics version 25.0 was used for statistical analysis. Group differences were assessed in a univariate analysis using Mann Whitney U (MWU) or Chi square test, respectively. Multivariate analysis of risk factors for treatment failure was performed using a binary logistic regression model including significant predictors from the univariate analysis.

\section{Results}

A total of 30 patients with belatacept conversion from a CNI-based immunosuppressive regimen at a median time of $127.5 \pm 91.3$ (range 99-190) months after transplantation were included. Table 1 shows patient and graft survival at 12 and 24 months after conversion to belatacept. Clinical characteristics at baseline and by status of graft failure or treatment failure, at 12 and 24 months are shown in Tables 2 and 3, respectively.

Median GFR at the time of conversion was $22.5 \pm 12$ (range 17-29.3) $\mathrm{mL} / \mathrm{min}$. In patients without graft loss GFR increased to 23.3 and $24.5 \mathrm{~mL} / \mathrm{min}$ at 12 and 24 months, respectively. Median GFR with imputation for graft loss was 21.5 and $18.5 \mathrm{~mL} / \mathrm{min}$ at $12 / 24$ months. As depicted in Table 1 and Fig. 1, eGFR slopes at 12 and 6 months before switch to belatacept were $\triangle$ GFR of $7.0 \pm 8.2$ and $-3.5 \pm 6.3 \mathrm{~mL} / \mathrm{min}$., respectively. At 12 and 24 months after switch eGFR slope flattened in patients 
Table 1 Patient survival, graft survival and renal function with and without imputation for missing values at 12 and 24 months after conversion to belatacept. GFR $9 \mathrm{~mL} / \mathrm{min}$ was imputed for kidney graft loss

\begin{tabular}{|c|c|c|c|c|}
\hline \multirow[b]{2}{*}{ Timepoint after switch } & \multicolumn{2}{|c|}{$\begin{array}{l}\text { Switch to belatacept } \\
(N=30) \\
\text { GFR at switch }=22.5 \pm 12 \mathrm{~mL} / \mathrm{min}\end{array}$} & \multicolumn{2}{|c|}{$\begin{array}{l}\text { Control cohort } \\
(N=56) \\
\text { GFR at start of observation }=24.5 \pm 14 \mathrm{~mL} / \mathrm{min}\end{array}$} \\
\hline & 12 months & 24 months & 12 months & 24 months \\
\hline Patient survival & $96.7 \%(29 / 30)$ & $90 \%(27 / 30)$ & $96.4 \%(54 / 56)$ & $91.07 \%(51 / 56)$ \\
\hline Death with functioning graft & $1 / 30$ & $3 / 30$ & $2 / 56$ & $5 / 56$ \\
\hline Kidney or pancreas graft loss & $6 / 30$ & $9 / 30$ & $8 / 56$ & $16 / 56$ \\
\hline Death-censored kidney graft survival & $79.3 \%(23 / 29)$ & $66.7 \%(18 / 27)$ & $85.2 \%(46 / 54)$ & $68.6 \%(35 / 51)$ \\
\hline Overall kidney graft survival & $76.7 \%(23 / 30)$ & $60.0 \%(18 / 30)$ & $82.1 \%(46 / 56)$ & $62.5 \%(35 / 56)$ \\
\hline Median GFR in patients without graft loss & $23.3 \pm 15$ & $24.5 \pm 15.0$ & $23.0 \pm 15$ & $24.0 \pm 9$ \\
\hline $\begin{array}{l}\Delta \text { GFR from GFR at baseline in patients } \\
\text { without graft loss }\end{array}$ & $1.3 \pm 5.9$ & $1.8 \pm 7.9$ & $-2.0 \pm 7.2$ & $-3.5 \pm 8.6$ \\
\hline Median GFR with imputation for graft loss & $21.5 \pm 18$ & $18.5 \pm 21$ & $21.5 \pm 18$ & $19 \pm 17$ \\
\hline $\begin{array}{l}\Delta \text { GFR from GFR at baseline in patients with } \\
\text { imputation for graft loss }\end{array}$ & $-0.56 \pm 6.8$ & $-1.54 \pm 8.4$ & $-3.3 \pm 7.6$ & $-5.5 \pm 8.3$ \\
\hline
\end{tabular}

Data were expressed as medians (interquartile range), means (standard deviation) or numbers

GFR glomerular filtration rate

with imputation for graft loss (mean $\triangle$ GFR of $-0.56 \pm$ 6.8 and $-1.54 \pm 8.4 \mathrm{~mL} / \mathrm{min}$, respectively) and increased in patients without imputation for graft loss (mean $\triangle$ GFR of $1.3 \pm 5.9$ and $1.8 \pm 7.9 \mathrm{~mL} / \mathrm{min})$.

$14 / 30(46.7 \%)$ patients were DSA-positive at the time of conversion. The median treatment duration on belatacept until graft loss, death or last follow-up was $29.5 \pm$ 27 (range 11.8-38.8) months.

A kidney transplant biopsy was performed at a median time of $5 \pm 11.3$ (range 1-12.3) months prior to belatacept conversion in all patients. Rejection-related biopsy scores were assessed in detail (Tables 2 and 3). Seven patients displayed lesions showing a Banff microvascular inflammation (MVI) sum score of $\geq 2$, one of these patients was DSA negative and did not display $\mathrm{C} 4 \mathrm{~d}+$ staining. Before conversion, $5 / 6$ patients received specific treatment for active antibodymediated rejection by plasmaphereses $(n=5 / 5)$, immunoglobulins $(n=3 / 5)$, bortezomib $(n=1 / 5)$, rituximab $(n=2 / 5)$, cyclophosphamide $(n=1 / 5)$ or thymoglobulin $(n=1 / 5)$.

\section{Patient survival at 12 and 24 months}

Patient survival at 12 months was $96.7 \%$ (29/30), as one 60-year old patient died from sudden death at 8 months. Patient survival at 24 months was $90 \%(27 / 30)$, as one 67 -year old patient died from central nervous system post-transplant lymphoproliferative disorder at month 22 , and one 63-year old patient died from intracranial bleeding at month 13 after conversion.

\section{DSA, rejection and graft survival at 12 and 24 months} During 24 months follow-up, no de novo DSA were found. However, of 14 patients with DSA-positivity at the time of conversion, one became DSA-negative after 12 months, and another after 24 months. Interestingly, both had been identified as nonadherent by the treating physicians prior to belatacept conversion and showed treatment response after 12 and 24 months.

Only one overt rejection episode occurred in a 39-year old female combined pancreas and kidney recipient after belatacept conversion. This was a severe pancreas graft rejection, refractory to thymoglobulin and steroid bolus therapy, which lead to graft loss at 4 months during follow up. Only one kidney transplant biopsy was taken after conversion, showing no signs of acute humoral or cellular rejection.

Overall graft survival at 1 year was $76.7 \%$ (23/30) including one death with functioning graft, 4 kidney transplant recipients and the 2 combined pancreas and kidney transplant patients who experienced renal or pancreas graft losses, respectively (Table 1). Overall renal graft survival at 24 months was $60.0 \%$ (18/30) including 3 deaths with functioning grafts and 9 renal or pancreas graft losses, respectively. Graft survival censored for death was $79.3 \%(23 / 29)$ at 12 months and $66.7 \%(18 / 27)$ at 24 months (Table 1$)$.

\section{Risk factors for renal graft loss at $\mathbf{1 2}$ and $\mathbf{2 4}$ months}

In the univariate analysis of risk factors for graft loss, GFR $<25 \mathrm{~mL} / \mathrm{min} \quad(p=0.042)$ and MVI sum score $\geq 2$ $(p=0.023)$ at conversion were significant at 24 months (Table 2). In binary logistic regression analyses models including GFR $<25 \mathrm{~mL} / \mathrm{min}$ at conversion and MVI sum score $\geq 2$, only MVI sum score $\geq 2$ was significant at 24 months $(p=0.038$, OR 13.0, 95\% CI 1.15-146.8, not shown). 
Table 2 Baseline characteristics in belatacept cohort by status for graft failure at 12 and 24 months censored for death

\begin{tabular}{|c|c|c|c|c|c|c|c|}
\hline Patient characteristics & $\begin{array}{l}\text { All patients } \\
(N=30)\end{array}$ & $\begin{array}{l}\text { Functioning graft at } \\
12 \text { months }(N=23)\end{array}$ & $\begin{array}{l}\text { Graft failure at } \\
12 \text { months }(N=6)\end{array}$ & $P$ value & $\begin{array}{l}\text { Functioning graft at } \\
24 \text { months }(N=18)\end{array}$ & $\begin{array}{l}\text { Graft failure at } \\
24 \text { months }(N=9)\end{array}$ & $P$ value \\
\hline Age (y) & $53.5 \pm 26$ & $54.0 \pm 19$ & $43 \pm 23$ & 0.302 & $51.5 \pm 23$ & $48 \pm 28$ & 0.940 \\
\hline Donor age (y) & $48.0 \pm 23$ & $50.0 \pm 26$ & $44 \pm 19$ & 0.555 & $45 \pm 26$ & $48.5 \pm 23$ & 0.825 \\
\hline Gender (m/f) & $20 / 10$ & $15 / 8$ & $4 / 2$ & 1.000 & $12 / 6$ & $5 / 4$ & 0.683 \\
\hline Post-transplant diabetes & $3 / 30$ & $3 / 23$ & $0 / 6$ & 1.000 & $3 / 18$ & 0/9 & 0.529 \\
\hline BMl & $25.3 \pm 4.1$ & $24.9 \pm 3.9$ & $25.4 \pm 6.6$ & 0.511 & $24.1 \pm 5.6$ & $25.5 \pm 5.6$ & 0.194 \\
\hline Systolic BP (mmHg) & $134 \pm 17$ & $138 \pm 20$ & $131.5 \pm 30$ & 0.384 & $136.5 \pm 21$ & $133 \pm 16$ & 0.348 \\
\hline Diastolic BP (mmHg) & $84 \pm 10$ & $84 \pm 10$ & $84 \pm 31$ & 0.581 & $86 \pm 11$ & $84 \pm 18$ & 0.691 \\
\hline Time after transplantation (m) & $127.5 \pm 91.3$ & $128 \pm 130$ & $127.5 \pm 52.5$ & 0.773 & $133.5 \pm 135.5$ & $126 \pm 35$ & 0.275 \\
\hline eGFR (mL/min) & $22.5 \pm 12$ & $24.0 \pm 13$ & $19.0 \pm 10$ & 0.302 & $25.5 \pm 12$ & $18.0 \pm 4$ & 0.095 \\
\hline eGFR $<25 \mathrm{~mL} / \mathrm{min}$ & $17 / 30$ & $12 / 23$ & $5 / 6$ & 0.354 & $8 / 18$ & $8 / 9$ & 0.042 \\
\hline Proteinuria (mg/g creatinine) & $840 \pm 1166$ & $830 \pm 950$ & $1256 \pm 3211$ & 0.302 & $657 \pm 1276$ & $869 \pm 2017$ & 0.668 \\
\hline Living donor transplants & $8 / 30$ & $6 / 23$ & $2 / 6$ & 1.000 & $4 / 18$ & $3 / 9$ & 0.653 \\
\hline pancreas/kidney & $2 / 30$ & - & - & & & & \\
\hline \multicolumn{8}{|l|}{ Immunosuppression } \\
\hline Tacrolimus & $22 / 30$ & $15 / 23$ & $6 / 6$ & 0.148 & $13 / 18$ & $6 / 9$ & 1.000 \\
\hline Cyclosporine A & $8 / 30$ & $8 / 23$ & $0 / 6$ & 0.148 & $5 / 18$ & $3 / 9$ & 1.000 \\
\hline Mycophenolic acid & $27 / 30$ & $20 / 23$ & $6 / 6$ & 1.000 & $16 / 18$ & 9/9 & 0.538 \\
\hline Azathioprin & $2 / 30$ & $2 / 23$ & $0 / 6$ & 1.000 & $1 / 18$ & 0/9 & 1.000 \\
\hline Steroid & $24 / 30$ & $17 / 23$ & $6 / 6$ & 0.295 & $13 / 18$ & $8 / 9$ & 0.628 \\
\hline DSA & $14 / 30$ & $11 / 23$ & $2 / 6$ & 0.663 & $8 / 18$ & $4 / 9$ & 1.000 \\
\hline h/o any rejection & $15 / 30$ & $10 / 23$ & $5 / 6$ & 0.169 & $7 / 18$ & $7 / 9$ & 0.103 \\
\hline aTCMR & $3 / 30$ & $1 / 23$ & $2 / 6$ & 0.100 & $1 / 18$ & $2 / 9$ & 0.250 \\
\hline aABMR & $6 / 30$ & $4 / 23$ & $2 / 6$ & 0.575 & $2 / 18$ & $4 / 9$ & 0.136 \\
\hline \multicolumn{8}{|l|}{ Biopsy scores } \\
\hline glomerular scarring (\%) & $30.5 \pm 33$ & $27 \pm 31$ & $42 \pm 27$ & 0.302 & $29 \pm 27$ & $32 \pm 43$ & 0.860 \\
\hline $\mathrm{cg}$ & $0.0 \pm 3.0$ & $0.0 \pm 3.0$ & $1.25 \pm 3.0$ & 0.694 & $0.0 \pm 1.1$ & $2.5 \pm 3.0$ & 0.232 \\
\hline $\mathrm{ct}$ & $1.0 \pm 1.0$ & $1.0 \pm 1.0$ & $1.0 \pm 2.0$ & 0.546 & $1.0 \pm 1.0$ & $1.0 \pm 1.0$ & 0.348 \\
\hline $\mathrm{ci}$ & $1.0 \pm 1.0$ & $1.0 \pm 1.0$ & $1.0 \pm 2.0$ & 0.477 & $1.0 \pm 1.0$ & $1.0 \pm 1.0$ & 0.253 \\
\hline $\mathrm{cV}$ & $2.0 \pm 2.0$ & $2.0 \pm 2.0$ & $1.5 \pm 1.0$ & 0.427 & $2.0 \pm 3.0$ & $2.0 \pm 1.0$ & 0.560 \\
\hline $\mathrm{mm}$ & $1.0 \pm 2.0$ & $1.0 \pm 1.0$ & $2.0 \pm 1.5$ & 0.071 & $0.0 \pm 1.0$ & $2.0 \pm 1.5$ & 0.067 \\
\hline ah & $3.0 \pm 0.0$ & $3.0 \pm 0.0$ & $3.0 \pm 0.3$ & 0.979 & $0.0 \pm 1.0$ & $3.0 \pm 1.0$ & 0.860 \\
\hline$g$ & $0.0 \pm 1.0$ & $0.0 \pm 1.0$ & $0.0 \pm 1.9$ & 0.694 & $0.0 \pm 1.0$ & $0.0 \pm 1.8$ & 0.668 \\
\hline t & $0.0 \pm 0.0$ & $0.0 \pm 0.0$ & $0.0 \pm 2.0$ & 0.384 & $0.0 \pm 0.0$ & $0.0 \pm 1.0$ & 0.322 \\
\hline i & $0.0 \pm 0.0$ & $0.0 \pm 0.0$ & $0.0 \pm 2.0$ & 0.546 & $0.0 \pm 0.0$ & $0.0 \pm 1.0$ & 0.781 \\
\hline$v$ & $0.0 \pm 0.0$ & $0.0 \pm 0.0$ & $0.0 \pm 0.0$ & 1.000 & $0.0 \pm 0.0$ & $0.0 \pm 0.0$ & 1.000 \\
\hline ptc & $0.0 \pm 0.0$ & $0.0 \pm 0.0$ & $0.5 \pm 3.0$ & 0.102 & $0.0 \pm 0.0$ & $0.0 \pm 0.0$ & 0.067 \\
\hline $\mathrm{C} 4 \mathrm{~d}$ & $0.0 \pm 0.0$ & $0.0 \pm 0.0$ & $0.0 \pm 1.0$ & 0.655 & $0.0 \pm 0.0$ & $0.0 \pm 0.0$ & 0.820 \\
\hline MVI sum score & $0.0 \pm 1.3$ & $0.0 \pm 1.0$ & $1.25 \pm 3.8$ & 0.254 & $0.0 \pm 0.0$ & $2.0 \pm 3.0$ & 0.095 \\
\hline MVI sum score $\geqq 2$ & $7 / 30$ & $4 / 23$ & $3 / 6$ & 0.131 & $2 / 18$ & $5 / 9$ & 0.023 \\
\hline
\end{tabular}

Data were expressed as medians (interquartile range), or numbers

$B M I$ body mass index, BP Blood pressure, eGFR estimated glomerular filtration rate, DSA donor specific antibodies, $h / o$ history of, aTCMR active T cell mediated rejection, $A A B M R$ active antibody-mediated rejection, $M V I$ microvascular inflammation 
Table 3 Baseline characteristics in belatacept cohort by status for treatment success or failure censored for death. Treatment failure was defined as graft failure or GFR deterioration compared to the time of conversion

\begin{tabular}{|c|c|c|c|c|c|c|c|}
\hline Patient characteristics & $\begin{array}{l}\text { All patients } \\
(N=30)\end{array}$ & $\begin{array}{l}\text { Treatment success at } \\
12 \text { months }(N=16)\end{array}$ & $\begin{array}{l}\text { Treatment failure at } \\
12 \text { months }(N=13)\end{array}$ & $P$ value & $\begin{array}{l}\text { Treatment success at } \\
24 \text { months }(N=12)\end{array}$ & $\begin{array}{l}\text { Treatment failure at } \\
24 \text { months }(N=15)\end{array}$ & $P$ value \\
\hline Age (y) & $53.5 \pm 26$ & $53.5 \pm 26$ & $50 \pm 29$ & 0.983 & $52 \pm 18$ & $50 \pm 30$ & 0.829 \\
\hline Donor age (y) & $48 \pm 23$ & $46.5 \pm 31$ & $46 \pm 22$ & 0.689 & $43 \pm 26$ & $52.5 \pm 23$ & 0.134 \\
\hline Gender (m/f) & $20 / 10$ & $11 / 5$ & $8 / 5$ & 0.714 & $10 / 2$ & $7 / 8$ & 0.107 \\
\hline Post-transplant diabetes & $3 / 30$ & $2 / 16$ & $1 / 13$ & 1.000 & $1 / 12$ & $2 / 15$ & 1.000 \\
\hline BMl & $25.3 \pm 4.1$ & $24.7 \pm 4.0$ & $25.5 \pm 6.1$ & 0.650 & $24.7 \pm 4.0$ & $25.3 \pm 7.9$ & 0.100 \\
\hline Systolic BP (mmHg) & $134 \pm 17$ & $139 \pm 24$ & $133 \pm 18$ & 0.199 & $139 \pm 27$ & $132 \pm 14$ & 0.183 \\
\hline Diastolic BP (mmHg) & $84 \pm 10$ & $85 \pm 10$ & $84 \pm 12$ & 0.812 & $88.5 \pm 12$ & $83 \pm 13$ & 0.300 \\
\hline Time after transplantation (m) & $127.5 \pm 91.3$ & $128.5 \pm 148.8$ & $127 \pm 60$ & 0.619 & $133.5 \pm 125.8$ & $126 \pm 74$ & 0.300 \\
\hline eGFR (mL/min) & $22.5 \pm 12$ & $25 \pm 12$ & $20 \pm 10$ & 0.449 & $25.5 \pm 14$ & $20.0 \pm 12$ & 0.126 \\
\hline $\mathrm{eGFR}<25 \mathrm{~mL} / \mathrm{min}$ & $17 / 30$ & $7 / 16$ & $10 / 13$ & 0.130 & $5 / 12$ & $11 / 15$ & 0.130 \\
\hline Proteinuria (mg/g creatinine) & $840 \pm 1166$ & $647 \pm 1125$ & $890 \pm 1857$ & 0.398 & $452 \pm 1125$ & $869 \pm 1592$ & 0.399 \\
\hline Living donor transplants & $8 / 30$ & $3 / 16$ & $5 / 13$ & 0.406 & $3 / 12$ & $4 / 15$ & 1.000 \\
\hline pancreas/kidney & $2 / 30$ & - & - & - & & & \\
\hline \multicolumn{8}{|l|}{ Immunosuppression } \\
\hline Tacrolimus & $22 / 30$ & $12 / 4$ & $9 / 4$ & 1.000 & $9 / 12$ & $10 / 15$ & 0.696 \\
\hline Cyclosporine A & $8 / 30$ & $4 / 12$ & $4 / 9$ & 1.000 & $3 / 12$ & $5 / 15$ & 0.696 \\
\hline Mycophenolic acid & $27 / 30$ & $13 / 3$ & $13 / 0$ & 0.232 & $10 / 12$ & $15 / 15$ & 0.188 \\
\hline Azathioprin & $2 / 30$ & $2 / 14$ & $0 / 13$ & 0.488 & $1 / 12$ & $0 / 15$ & 0.444 \\
\hline Steroid & $24 / 30$ & $13 / 3$ & $10 / 3$ & 1.000 & $10 / 12$ & $11 / 15$ & 0.662 \\
\hline DSA & $14 / 30$ & $7 / 16$ & $6 / 13$ & 1.000 & $5 / 12$ & $7 / 15$ & 1.000 \\
\hline h/o any rejection & $15 / 30$ & $6 / 16$ & $9 / 13$ & 0.139 & $6 / 12$ & $8 / 15$ & 1.000 \\
\hline aTCMR & $3 / 30$ & $1 / 16$ & $2 / 13$ & 0.573 & $1 / 12$ & $2 / 15$ & 1.000 \\
\hline aABMR & $6 / 30$ & $1 / 16$ & $5 / 13$ & 0.064 & $1 / 12$ & $5 / 15$ & 0.182 \\
\hline \multicolumn{8}{|l|}{ Biopsy scores } \\
\hline Glomerular scarring (\%) & $30.5 \pm 33$ & $26 \pm 29$ & $32 \pm 35.5$ & 0.423 & $27 \pm 33$ & $32 \pm 36$ & 0.829 \\
\hline $\mathrm{Cg}$ & $0.0 \pm 3.0$ & $0.0 \pm 2.6$ & $1.0 \pm 3.0$ & 0.288 & $0.0 \pm 1.1$ & $1.0 \pm 3.0$ & 0.236 \\
\hline $\mathrm{ct}$ & $1.0 \pm 1.0$ & $1.0 \pm 1.0$ & $1.0 \pm 2.0$ & 0.983 & $1.0 \pm 1.0$ & $1.0 \pm 1.0$ & 0.548 \\
\hline $\mathrm{ci}$ & $1.0 \pm 1.0$ & $1.0 \pm 1.0$ & $1.0 \pm 1.0$ & 0.846 & $1.0 \pm 1.0$ & $1.0 \pm 1.0$ & 0.683 \\
\hline $\mathrm{cV}$ & $2.0 \pm 2.0$ & $2.0 \pm 2.0$ & $2.0 \pm 2.0$ & 0.339 & $2.0 \pm 3.0$ & $2.0 \pm 1.0$ & 0.959 \\
\hline $\mathrm{mm}$ & $1.0 \pm 2.0$ & $0.0 \pm 1.8$ & $1.0 \pm 1.5$ & 0.170 & $0.0 \pm 1.8$ & $1.0 \pm 2.0$ & 0.373 \\
\hline ah & $3.0 \pm 0.0$ & $3.0 \pm 0.0$ & $3.0 \pm 0.5$ & 0.650 & $3.0 \pm 0.4$ & $3.0 \pm 0.0$ & 0.581 \\
\hline g & $0.0 \pm 1.0$ & $0.0 \pm 0.8$ & $0.0 \pm 1.8$ & 0.589 & $0.0 \pm 0.8$ & $0.0 \pm 1.5$ & 0.581 \\
\hline $\mathrm{t}$ & $0.0 \pm 0.0$ & $0.0 \pm 0.0$ & $0.0 \pm 1.0$ & 0.589 & $0.0 \pm 0.0$ & $0.0 \pm 0.0$ & 0.829 \\
\hline i & $0.0 \pm 0.0$ & $0.0 \pm 0.0$ & $0.0 \pm 1.0$ & 0.156 & $0.0 \pm 0.0$ & $0.0 \pm 0.0$ & 0.905 \\
\hline$v$ & $0.0 \pm 0.0$ & $0.0 \pm 0.0$ & $0.0 \pm 0.0$ & 1.000 & $0.0 \pm 0.0$ & $0.0 \pm 0.0$ & 1.000 \\
\hline ptc & $0.0 \pm 0.0$ & $0.0 \pm 0.0$ & $0.0 \pm 2.0$ & 0.170 & $0.0 \pm 0.0$ & $0.0 \pm 1.0$ & 0.256 \\
\hline$C 4 d$ & $0.0 \pm 0.0$ & $0.0 \pm 0.0$ & $0.0 \pm 0.0$ & 0.948 & $0.0 \pm 0.0$ & $0.0 \pm 0.0$ & 0.981 \\
\hline MVI sum score & $0.0 \pm 1.3$ & $0.0 \pm 0.8$ & $0.0 \pm 2.8$ & 0.144 & $0.0 \pm 0.8$ & $0.0 \pm 2.5$ & 0.183 \\
\hline MVI sum score $\geqq 2$ & $7 / 30$ & $1 / 16$ & $6 / 13$ & 0.026 & $1 / 12$ & $6 / 15$ & 0.091 \\
\hline
\end{tabular}

Data were expressed as medians (interquartile range), or numbers

$B M I$ body mass index, $B P$ blood pressure, eGFR estimated glomerular filtration rate, DSA donor specific antibodies, $h / o$ history of, aTCMR active T cell mediated rejection, $a A B M R$ active antibody-mediated rejection, $M V I$ microvascular inflammation 


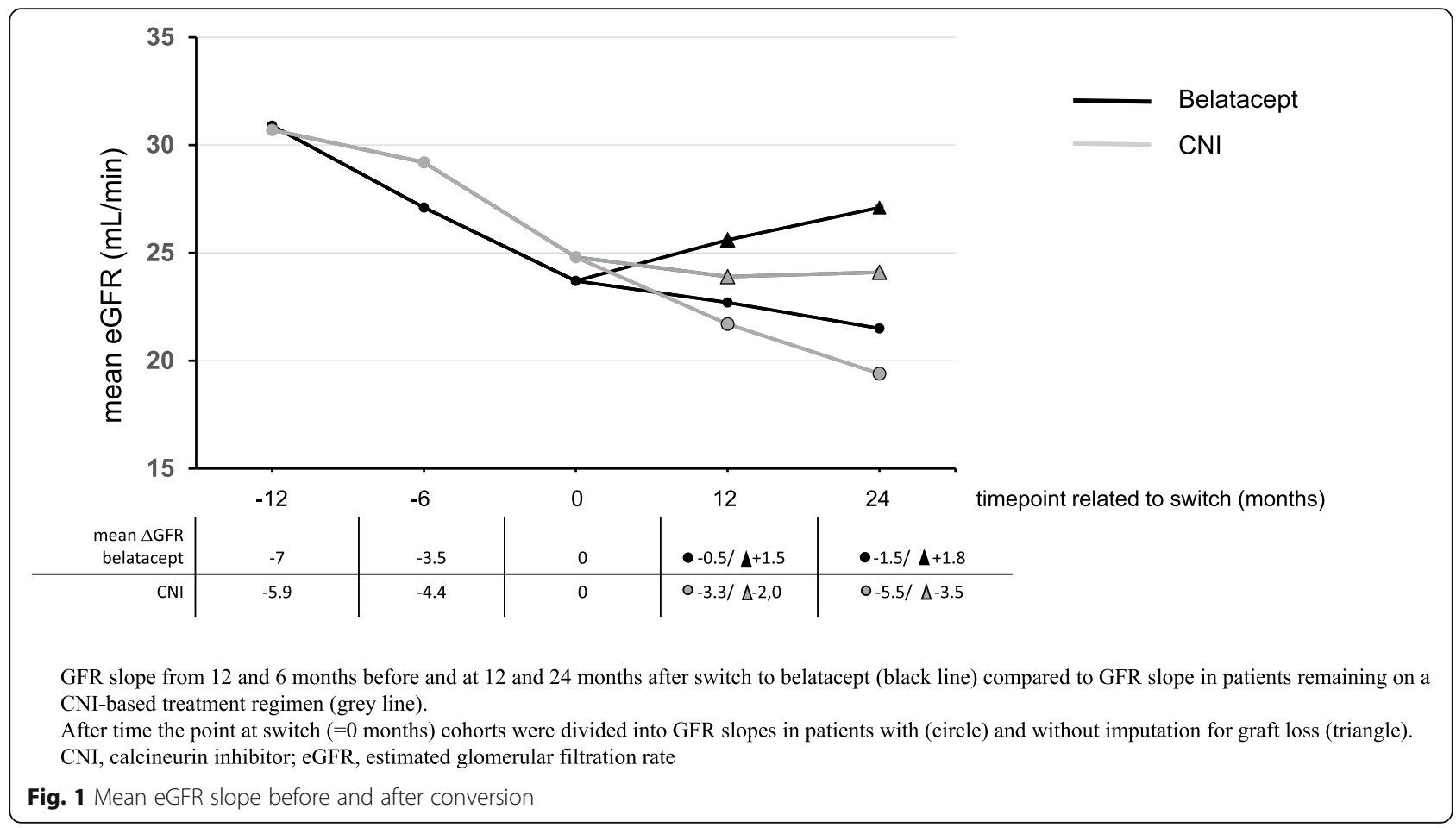

Noteworthy, DSA-positivity at time of conversion was not associated with graft loss at 12 or 24 months.

\section{Risk factors for treatment failure at 12 and 24 months}

In the univariate analysis of risk factors for treatment failure, only MVI sum score $\geq 2$ was significant ( $p=$ 0.023 ) at 12 months (Table 3 ). In binary logistic regression analysis models including GFR $<25 \mathrm{~mL} / \mathrm{min}$ at conversion and MVI sum score $\geq 2$, only MVI sum score $\geq 2$ was significant at 12 months $(p=0.037$, OR $13.2,95 \% \mathrm{CI}$ 1.17-147.8, not shown).

Again, DSA-positivity was not associated with treatment failure at 12 or 24 months.

\section{Control cohort \\ Characteristics and outcome}

We used a control cohort of 56 patients with CNIbased maintenance therapy to compare findings within the belatacept cohort for graft survival, outcome and renal function during the same observation period. Table 4 shows demographic and baseline characteristics of both cohorts. There were no significant differences e.g. regarding age, donor age, gender, time after transplantation, eGFR at switch, immunosuppression. Furthermore, except for arterial hyalinosis, no significant differences were observed regarding active and chronic lesions within renal transplant biopsies.

Cases with DSA positivity (19 out of 56), and an MVI sum score $\geq 2(8 / 56)$, were fewer in the control cohort compared to the belatacept cohort, while similar rates of active T-cell-mediated and active antibody-mediated rejections (aABMR) were observed in both groups. Eleven out of twelve aABMR received specific treatment prior to the start of observation time point ( 10 by plasmaphereses, 10 with immunoglobulins, 1 bortezomib, 7 with rituximab, 1 with cyclophosphamide). While eGFR slopes at 12 and 6 months prior to "virtual" switch were similar to eGFR slopes in belatacept patients (mean $\triangle$ GFR of $-5.9 \pm$ 7.0 and $-4.4 \pm 6.6 \mathrm{~mL} / \mathrm{min}$, respectively), eGFR slope did not flatten during 12 and 24 months follow up (mean $\triangle$ GFR of $-3.3 \pm 7.6$ and $-5.5 \pm 8.3$ ) in patients with imputation for graft loss. Moreover, in contrast to the belatacept group, eGFR did not increase in patients without imputation for graft loss at 12 and 24 months (mean $\triangle$ GFR of $-2.0 \pm 7.2$ and $-3.5 \pm 8.6 \mathrm{~mL} /$ min, respectively). Results are shown in Table 1 . Baseline characteristics by status for graft failure at 12 and 24 months censored for death are presented in the Additional file 1.

Notably, fewer patients in the control cohort had stable or improved renal function after 24 months (12/ 30 in belatacept patients versus $13 / 56$ in control patients, Additional file 2).

\section{Discussion}

Increasing numbers of studies reported the feasibility and safety of late switch to belatacept in certain patient groups, but still few is known on the benefit of belatacept-based treatment in patients with higher 
Table 4 Demographic and Clinical Characteristics of Belatacept and Control patients

\begin{tabular}{|c|c|c|c|}
\hline Cohort characteristics & Belatacept $(N=30)$ & $\mathrm{CNI}(\mathrm{N}=56)$ & $P$ value \\
\hline Age (y) & $53.5 \pm 26$ & $52.0 \pm 21$ & 0.700 \\
\hline Donor age (y) & $48.0 \pm 23$ & $46.0 \pm 23$ & 0.907 \\
\hline Gender (m/f) & $20 / 10$ & $19 / 37$ & 0.956 \\
\hline Post-transplant diabetes & $3 / 30$ & $5 / 56$ & 0.871 \\
\hline BMI & $25.3 \pm 4.1$ & $22.9 \pm 8.3$ & 0.213 \\
\hline Systolic BP (mmHg) & $134 \pm 17$ & $130 \pm 51$ & 0.076 \\
\hline Diastolic BP (mmHg) & $84 \pm 10$ & $80 \pm 13$ & 0.054 \\
\hline Time after transplantation (m) & $127.5 \pm 91.3$ & $113.0 \pm 102.5$ & 0.500 \\
\hline eGFR (mL/min) & $22.5 \pm 12$ & $24.5 \pm 14$ & 0.583 \\
\hline $\mathrm{eGFR}<25 \mathrm{~mL} / \mathrm{min}$ & $17 / 30$ & $28 / 56$ & 0.667 \\
\hline Proteinuria (mg/g creatinine) & $840 \pm 1166$ & $280.5 \pm 1270$ & 0.157 \\
\hline Living donor transplants & $8 / 30$ & $8 / 56$ & \\
\hline Pancreas/kidney & $2 / 30$ & $6 / 56$ & \\
\hline \multicolumn{4}{|l|}{ Immunosuppression } \\
\hline Tacrolimus & $22 / 30$ & $42 / 56$ & 0.867 \\
\hline Cyclosporine A & $8 / 30$ & $14 / 56$ & 0.867 \\
\hline Mycophenolic acid & $27 / 30$ & $54 / 56$ & 0.227 \\
\hline Azathioprin & $2 / 30$ & $0 / 56$ & 0.052 \\
\hline Steroid & $24 / 30$ & $45 / 56$ & 0.969 \\
\hline DSA & $14 / 30$ & $19 / 56$ & 0.336 \\
\hline h/o any rejection & $15 / 30$ & $20 / 56$ & 0.201 \\
\hline aTCMR & $3 / 30$ & $7 / 56$ & 0.732 \\
\hline aABMR & $6 / 30$ & $12 / 56$ & 0.990 \\
\hline Histology before switch (m) & $5 \pm 11.3$ & $9.5 \pm 26.5$ & 0.236 \\
\hline \multicolumn{4}{|l|}{ Biopsy scores } \\
\hline glomerular scarring (\%) & $30.5 \pm 33$ & $28.5 \pm 45$ & 0.942 \\
\hline $\mathrm{cg}$ & $0.0 \pm 3.0$ & $0.0 \pm 1.4$ & 0.202 \\
\hline $\mathrm{ct}$ & $1.0 \pm 1.0$ & $1.0 \pm 1.0$ & 1.000 \\
\hline $\mathrm{Ci}$ & $1.0 \pm 1.0$ & $1.0 \pm 1.0$ & 0.705 \\
\hline$c \mathrm{~V}$ & $2.0 \pm 2.0$ & $1.0 \pm 1.0$ & 0.368 \\
\hline $\mathrm{mm}$ & $1.0 \pm 2.0$ & $1.0 \pm 2.0$ & 0.845 \\
\hline ah & $3.0 \pm 0.0$ & $2.75 \pm 1.0$ & 0.003 \\
\hline g & $0.0 \pm 1.0$ & $0.0 \pm 0.0$ & 0.307 \\
\hline $\mathrm{t}$ & $0.0 \pm 0.0$ & $0.0 \pm 0.0$ & 0.865 \\
\hline i & $0.0 \pm 0.0$ & $0.0 \pm 1.0$ & 0.243 \\
\hline$v$ & $0.0 \pm 0.0$ & $0.0 \pm 0.0$ & 0.129 \\
\hline ptc & $0.0 \pm 0.0$ & $0.0 \pm 0.0$ & 0.956 \\
\hline diffuse ptc (y/n) & $0 / 30$ & $3 / 56$ & 0.333 \\
\hline$c 4 d$ & $0.0 \pm 0.0$ & $0.0 \pm 0.0$ & 1.000 \\
\hline MVI sum score & $0.0 \pm 1.3$ & $0.0 \pm 1.0$ & 0.448 \\
\hline MVI sum score $\geqq 2$ & $7 / 30$ & $8 / 56$ & 0.295 \\
\hline
\end{tabular}

Data were expressed as medians (interquartile range), or numbers

$B M I$ body mass index, BP blood pressure, eGFR estimated glomerular filtration rate, DSA donor specific antibodies, $h / o$ history of, aTCMR active T cell mediated rejection, $a A B M R$ active antibody-mediated rejection, $M V I$ microvascular inflammation 
immunological risk for both the de novo and switch situations. Patient populations are often very heterogenous with different time points or treatment strategies before switch to belatacept. In our study we strictly focused on the switch of patients from CNI-based treatment to belatacept in the very late phase after transplantation (median of $10.6 \pm 7.6$ years).

The development of donor-specific antibodies (DSA) and subsequent antibody-mediated rejection (ABMR) are key factors for late allograft failure $[19,20]$. While the percentage of patients with DSA in larger analyses was $20 \%$ by 5 years after transplantation [21], in our study a much higher percentage of $46.7 \%$ had DSA at the time of conversion.

However, we found no association of DSA positivity with graft loss or treatment failure at 12 or 24 months after conversion. Rather, we found an association of significant microvascular inflammation (MVI) score in biopsies prior to conversion to belatacept with treatment failure at 24 months, defined by glomerulitis (g) and peritubular capillaritis (ptc) $\geq 2$, which is part of the histopathological criteria for diagnosis of active ABMR [17]. MVI has been validated in molecular studies and closely associated with outcome, even without C4d deposition or DSA [22-27].

Our data suggest that not DSA positivity per se, but the presence of active microvascular inflammation, which is suspicious, but not restricted to active humoral rejection, is a risk factor for patients that are converted from a CNI-based regimen to belatacept treatment.

Recently, a single center study on de novo belatacept use in a real life scenario including re-transplant patients, patients with higher panel reactive antibodies und HLA mismatches and higher percentages of "Afro American" patients found significantly increased acute rejection rates including more severe rejection grades with belatacept versus tacrolimus, ultimately leading to a modification of the immunosuppressive protocol including combined use of tacrolimus tapering until 9-12 months after transplantation [28].

Using thymoglobulin induction and belatacept in 49 patients with preformed DSA of mild mean fluorescence intensities ( $\max 500-3000$ ), Leibler et al. found no ABMR after 12 months, albeit again with significantly increased rates of T-cell-mediated rejections with an incidence of $25.4 \%$ [29].

In line with the previous phase II study by Rostaing et al., who converted patients between 6 and 36 months after kidney transplantation to belatacept, albeit with stable function, we included only patients with prolonged exposure to the calcineurin inhibitors tacrolimus or cyclosporine [12]. At 1 and 3 years, a significant improvement in kidney function compared with cyclosporine was found [9]. Acute rejection rates in belatacept-treated patients cumulated at $8.3 \%$ after 36 months [9]. Darres et al. described the results of conversion to belatacept in 219 kidney transplants including 35 (16\%) patients with DSA and 9\% patients with mTORbased therapy from 5 European centers between 0 and 337 (mean 44) months after transplantation. Compared to our data, patients were converted earlier and displayed a higher eGFR at the time of conversion. Indication for conversion was mostly impaired kidney function but also intolerance to CNI or mTOR inhibitors. Graft loss occurred in $11 \%$ of patients at the end of follow up, and belatacept was stopped for other reasons in another $11 \%$ of patients. In the remaining cohort, eGFR increased from 32 to 38 $\mathrm{mL} / \mathrm{min}$, with the highest increase in patients switched before month 3 posttransplant. After conversion to belatacept $8.2 \%$ of patients developed an acute rejection episode, and 3 patients developed DSA. The authors concluded that overall efficacy and safety were good, even in patients with DSA [14].

In our study, DSA positivity was not associated with graft or treatment failure at 12 and 24 months, only one overt pancreas graft rejection (3.3\%) occurred leading to graft loss at 4 months after conversion. Interestingly, no patient developed de novo DSA after belatacept conversion.

One limit of our study is the lack of follow-up biopsies on patients with graft deterioration, as only one followup biopsy was performed, showing no rejection.

Dürr et al. reported the conversion of 69 renal transplant patients including $20 \%$ patients under mTOR inhibitors to belatacept at a mean time of 68.8 months after transplantation. 38\% showed a significant eGFR increase after 12 months [15]. Notably, DSA mean fluorescence intensities after conversion as well as higher proteinuria before conversion associated with nonresponder status after 12 months. In contrast to the present study, higher proteinuria at the time of conversion was associated with less GFR increase. This might be related to exclusion of patients with mTOR-inhibitorbased treatment in our present study.

Notably, in our study both patients with combined pancreas and renal grafts experienced graft losses during 24 months follow up. Beside the pancreas graft loss due to rejection, the other patient lossed his kidney graft due to unknown reasons. Few data are available on switching pancreas transplant patients to belatacept. A previous study reported successful conversion of two patients from tacrolimus to belatacept and sirolimus [30]. Furthermore, belatacept together with sirolimus has been successfully used in rhesus monkeys with islet transplantation [31]. Thus, more data are needed to evaluate safety and efficacy in these patients.

To draw stronger conclusions about the results of our study, we compared the belatacept group with a control 
cohort under CNI maintenance therapy. We could demonstrate comparable outcome results regarding death and graft survival, albeit no better outcome regarding renal function. Compared to controls, patients in the belatacept group slowed loss of renal function after conversion during 12 and 24 months follow up and moreover increased GFR in the analysis in cases without imputation for graft loss. However, due to the retrospective nature of our study, data are biased and interpretation of differences between both groups has to be done cautiously. An ongoing large prospective, randomized trial will provide more robust data on the outcome of maintenance kidney transplant recipients following conversion to belatacept (NCT 01820572).

The limitations of our study are the small sample size limiting significant findings, albeit we included a high proportion of patients at immunological risk. The follow-up time for our study was still short, and belatacept may be more effective in preserving eGFR beyond 2 years after conversion. But due to a very late belatacept switch after transplantation with a higher risk of terminal transplant failure longer follow up might be difficult to interpret.

\section{Conclusions}

Rescue therapy with conversion from CNI to belatacept is feasible in patients with worsening renal function, even many years after transplantation and in DSApositive patients. However, patients at immunological risk such as those with combined pancreas and renal transplants, history of rejection, significant microvascular inflammation in biopsy and patients with severe graft impairment should be treated with caution and have a more in-depth evaluation in controlled studies to define the benefit of a conversion to belatacept to avoid rejection and graft losses.

\section{Supplementary information}

Supplementary information accompanies this paper at https://doi.org/10. 1186/s12882-020-01992-6.

Additional file 1. Baseline characteristics of Control cohort by status for graft failure at 12 and 24 months censored for death.

Additional file 2. Baseline characteristics of Control cohort by status for treatment success or failure censored for death.

\begin{abstract}
Abbreviations
aABMR: Active antibody-mediated rejection; aTCMR: Active T-cell-mediated rejection; BMI: Body mass index; CNI: Calcineurin inhibitor; DSA: Donorspecific antibody; GFR: Glomerular filtration rate; KTR: Kidney transplant recipient; mTOR: Mammalian target of rapamycin; MVI: Banff microvascular inflammation
\end{abstract}

Acknowledgements

Not applicable.

\section{Authors' contributions}

$M C$ and PN initiated, designed the study, collected and analyzed data and were major contributors in writing the manuscript. KB interpreted the data for analysis and was a major contributor revising the manuscript. FB, KW, NL, $D S, A K, S B, M D, A K$, and KUE collected and analyzed data and interpreted the data for analysis. All authors read and approved the final manuscript.

Funding

Open access funding provided by Projekt DEAL.

\section{Availability of data and materials}

The data generated and used in the analysis of this study are included in this published article. Additional data is available from the authors upon reasonable request.

\section{Ethics approval and consent to participate}

This study was approved by the institutional review board of the ethics committee of Charité Universitätsmedizin Berlin, Germany (approval number EA1/252/17). Written informed consent was obtained from all patients in a form accepted by the mentioned committee.

Consent for publication

Not applicable.

\section{Competing interests}

$M C$ received travel grants from Astellas.

MD received travel grants and speakers honoraria from BMS, Novartis, Roche and Astellas.

$\mathrm{KB}$ received research funds and/or honoraria from Abbvie, Alexion, Astellas, Bristol-Myers Squibb, Chiesi, Fresenius, Genentech, Hexal, Novartis, Otsuka, Pfizer, Roche, Siemens, and Veloxis Pharma.

SB received honoraria from Astellas, Bristol-Myers Squibb, Chiesi, Chilis, Novartis, Pfizer, and Roche.

$F B, K W, N L, D S, A K, K U E, P N$ have no conflict of interest to declare.

\section{Author details}

${ }^{1}$ Department of Nephrology and Medical Intensive Care, Charité Universitätsmedizin Berlin, Augustenburger Platz 1, 13353 Berlin, Germany. ${ }^{2}$ Department of Pathology, Charité Universitätsmedizin Berlin, Berlin, Germany. ${ }^{3}$ Tissue Typing Laboratory, Charité Universitätsmedizin Berlin, Berlin, Germany.

Received: 6 March 2020 Accepted: 29 July 2020

Published online: 20 August 2020

\section{References}

1. Nankivell BJ, Borrows RJ, Fung CL, O'Connell PJ, Allen RD, Chapman JR. The natural history of chronic allograft nephropathy. N Engl J Med. 2003;349(24): 2326-33.

2. Gaston RS. Chronic calcineurin inhibitor nephrotoxicity: reflections on an evolving paradigm. Clin J Am Soc Nephrol. 2009:4(12):2029-34.

3. Vincenti F, Larsen CP, Alberu J, Bresnahan B, Garcia VD, Kothari J, et al. Three-year outcomes from BENEFIT, a randomized, active-controlled, parallel-group study in adult kidney transplant recipients. Am J Transplant. 2012;12(1):210-7.

4. Vincenti F. Belatacept and long-term outcomes in kidney transplantation. N Engl J Med. 2016;374(26):2600-1.

5. Durrbach A, Pestana JM, Florman S, Del Carmen RM, Rostaing L, Kuypers D, et al. Long-term outcomes in Belatacept- versus cyclosporine-treated recipients of extended criteria donor kidneys: final results from BENEFIT-EXT, a phase III randomized study. Am J Transplant. 2016;16(11):3192-201.

6. Vincenti F, Blancho G, Durrbach A, Grannas G, Grinyo J, Meier-Kriesche HU, et al. Ten-year outcomes in a randomized phase II study of kidney transplant recipients administered belatacept 4-weekly or 8-weekly. Am J Transplant. 2017:17(12):3219-27.

7. Leibler C, Matignon M, Pilon C, Montespan F, Bigot J, Lang P, et al. Kidney transplant recipients treated with belatacept exhibit increased naive and transitional B cells. Am J Transplant. 2014;14(5):1173-82.

8. Leibler C, Thiolat A, Henique C, Samson C, Pilon C, Tamagne M, et al. Control of humoral response in renal transplantation by Belatacept depends 
on a direct effect on B cells and impaired $T$ follicular helper-B cell crosstalk. J Am Soc Nephrol. 2018;29(3):1049-62.

9. Grinyo JM, Del Carmen RM, Alberu J, Steinberg SM, Manfro RC, Nainan G, et al. Safety and efficacy outcomes 3 years after switching to Belatacept from a Calcineurin inhibitor in kidney transplant recipients: results from a phase 2 randomized trial. Am J Kidney Dis. 2017:69(5):587-94.

10. Nair V, Liriano-Ward L, Kent R, Huprikar S, Rana M, Florman SS, et al. Early conversion to belatacept after renal transplantation. Clin Transpl. 2017;31:5).

11. Le Meur Y, Aulagnon F, Bertrand D, Heng AE, Lavaud S, Caillard S, et al. Effect of an early switch to Belatacept among Calcineurin inhibitorintolerant graft recipients of kidneys from extended-criteria donors. Am J Transplant. 2016;16(7):2181-6.

12. Rostaing L, Massari P, Garcia VD, Mancilla-Urrea E, Nainan G, del Carmen RM, et al. Switching from calcineurin inhibitor-based regimens to a belataceptbased regimen in renal transplant recipients: a randomized phase II study. Clin J Am Soc Nephrol. 2011;6(2):430-9.

13. Brakemeier S, Kannenkeril D, Durr M, Braun T, Bachmann F, Schmidt D, et al. Experience with belatacept rescue therapy in kidney transplant recipients. Transpl Int. 2016;29(11):1184-95.

14. Darres A, Ulloa C, Brakemeier S, Garrouste C, Bestard O, Del Bello A, et al. Conversion to Belatacept in maintenance kidney transplant patients: a retrospective multicenter European study. Transplantation. 2018;102(9): 1545-52.

15. Durr M, Lachmann N, Zukunft B, Schmidt D, Budde K, Brakemeier S. Late conversion to Belatacept after kidney transplantation: outcome and prognostic factors. Transplant Proc. 2017;49(8):1747-56 e1741.

16. Gupta S, Rosales I, Wojciechowski D. Pilot analysis of late conversion to Belatacept in kidney transplant recipients for biopsy-proven chronic tacrolimus toxicity. J Transp Secur. 2018;2018:1968029.

17. Loupy A, Haas M, Solez K, Racusen L, Glotz D, Seron D, et al. The Banff 2015 kidney meeting report: current challenges in rejection classification and prospects for adopting molecular pathology. Am J Transplant. 2017;17(1):28-41.

18. Fritsche L, Schroter K, Lindemann G, Kunz R, Budde K, Neumayer HH. A web-based electronic patient record system as a means for collection of clinical data. Lect Notes Comput Sc. 1933;2000:198-205.

19. Einecke G, Sis B, Reeve J, Mengel M, Campbell PM, Hidalgo LG, et al. Antibody-mediated microcirculation injury is the major cause of late kidney transplant failure. Am J Transplant. 2009;9(11):2520-31.

20. Sellares J, de Freitas DG, Mengel M, Reeve J, Einecke G, Sis B, et al. Understanding the causes of kidney transplant failure: the dominant role of antibody-mediated rejection and nonadherence. Am J Transplant. 2012; 12(2):388-99.

21. Everly MJ, Rebellato LM, Haisch CE, Ozawa M, Parker K, Briley KP, et al. Incidence and impact of de novo donor-specific alloantibody in primary renal allografts. Transplantation. 2013;95(3):410-7.

22. de Kort H, Willicombe M, Brookes P, Dominy KM, Santos-Nunez E, Galliford JW, et al. Microcirculation inflammation associates with outcome in renal transplant patients with de novo donor-specific antibodies. Am J Transplant. 2013;13(2):485-92.

23. Gupta A, Broin PO, Bao Y, Pullman J, Kamal L, Ajaimy M, et al. Clinical and molecular significance of microvascular inflammation in transplant kidney biopsies. Kidney Int. 2016;89(1):217-25.

24. Kozakowski N, Herkner H, Bohmig GA, Regele H, Kornauth C, Bond G, et al. The diffuse extent of peritubular capillaritis in renal allograft rejection is an independent risk factor for graft loss. Kidney Int. 2015; 88(2):332-40.

25. Kozakowski N, Herkner H, Eskandary F, Eder M, Winnicki W, Klager J, et al. An integrative approach for the assessment of peritubular capillaritis extent and score in low-grade microvascular inflammationassociations with transplant glomerulopathy and graft loss. Nephrol Dial Transplant. 2019;34(1):166-74.

26. Loupy A, Hill GS, Suberbielle C, Charron D, Anglicheau D, Zuber J, et al. Significance of C4d Banff scores in early protocol biopsies of kidney transplant recipients with preformed donor-specific antibodies (DSA). Am J Transplant. 2011;11(1):56-65.

27. Sis B, Jhangri GS, Riopel J, Chang J, de Freitas DG, Hidalgo L, et al. A new diagnostic algorithm for antibody-mediated microcirculation inflammation in kidney transplants. Am J Transplant. 2012;12(5):1168-79.

28. Adams AB, Goldstein J, Garrett C, Zhang R, Patzer RE, Newell KA, et al. Belatacept combined with transient Calcineurin inhibitor therapy prevents rejection and promotes improved long-term renal allograft function. Am J Transplant. 2017;17(11):2922-36.

29. Leibler C, Matignon M, Moktefi A, Samson C, Zarour A, Malard S, et al. Belatacept in renal transplant recipient with mild immunologic risk factor: a pilot prospective study (BELACOR). Am J Transplant. 2019;19(3):894-906.

30. Mujtaba MA, Sharfuddin AA, Taber T, Chen J, Phillips CL, Goble M, et al. Conversion from tacrolimus to belatacept to prevent the progression of chronic kidney disease in pancreas transplantation: case report of two patients. Am J Transplant. 2014;14(11):2657-61.

31. Lowe MC, Badell IR, Turner AP, Thompson PW, Leopardi FV, Strobert EA, et al. Belatacept and sirolimus prolong nonhuman primate islet allograft survival: adverse consequences of concomitant alefacept therapy. Am J Transplant. 2013;13(2):312-9.

\section{Publisher's Note}

Springer Nature remains neutral with regard to jurisdictional claims in published maps and institutional affiliations.

\section{Ready to submit your research? Choose BMC and benefit from:}

- fast, convenient online submission

- thorough peer review by experienced researchers in your field

- rapid publication on acceptance

- support for research data, including large and complex data types

- gold Open Access which fosters wider collaboration and increased citations

- maximum visibility for your research: over $100 \mathrm{M}$ website views per year

At BMC, research is always in progress.

Learn more biomedcentral.com/submissions 